\title{
La stratégie eHealth en Valais
}

\section{Alex Gnaegia, \\ Philippe Wieser ${ }^{b}$, \\ Georges Dupuisc}

a Dr, Médecin-chef, Service d'informatique médicale et administrative, Institut Central des Hôpitaux Valaisans, Réseau Santé Valais

b Prof., Ecole Polytechnique Fédérale, Lausanne

c Dr, Médecin cantonal, Service de la santé publique, Sion

Les auteurs certifient qu'aucun conflit d'intérêt n'est lié à cet article.

Correspondance:

Dr Alex Gnaegi

Service d'informatique médicale et administrative

Institut Central des Hôpitaux Valaisans

Réseau Santé Valais

Av. Grand-Champsec 86

CH-1950 Sion

alex.gnaegi@ichv.ch

\section{Système d'information sanitaire}

L'Etat du Valais et plus précisément le service de la santé publique souhaite disposer de données de synthèse (anonymes) en quasi-temps réel, de manière à pouvoir établir des tableaux de bord efficaces et performants destinés, en fonction de ses objectifs stratégiques, à piloter et à coordonner la politique de santé de l'Etat. Dans ce but, le service de la santé, sur recommandation de 2 rapports d'experts [1,2 ] a initié en 2001 la constitution d'un Observatoire valaisan de la santé [3] chargé de collecter et analyser les données relatives au système sanitaire valaisan. Parallèlement un projet de mise en place d'un système d'information sanitaire a été amorcé. Celui-ci est composé de trois volets correspondants aux 3 grands composants de l'appareil sanitaire:

1. Les hôpitaux

2. Les établissements médico-sociaux et les centres de soins à domicile

3. La médecine ambulatoire

Le système d'information sanitaire doit ainsi fournir les données de synthèse pour l'autorité sanitaire mais également améliorer la qualité de soins en facilitant l'accès à l'information pour les professionnels de la santé.

\subsection{Hôpitaux projet Infoval}

Le concept d'informatisation des hôpitaux valaisans (projet Infoval) est articulé autour de quatre éléments clés (fig. 1): un système d'information administratif (admission des patients, facturation, comptabilité, etc.), un système d'information clinique (dossier patient informatisé DPI), un serveur d'identité et, finalement, un datawarehouse permettant de regrouper l'ensemble de l'information «anonymisée», médicale et administrative, à des fins de gestion, de stratégie, de planification, de suivi-contrôle ainsi qu'à toute étude et recherche épidémiologique.

\subsubsection{Dossier patient informatisé du Réseau Santé Valais}

Basé sur un progiciel commercial (Phoenix ${ }^{\circledast}$ de MCS Parametrix Solutions $A G^{\circledR}$ ), le dossier patient informatisé (DPI) a été implémenté progressivement entre 2002 et 2005 sur l'ensemble des services hospitaliers somatiques et psychiatriques du Réseau Santé Valais (RSV). Il est actuellement utilisé avec satisfaction [4] par quelques 2500 utilisateurs. Préalablement à la paramétrisation du logiciel, une phase de standardisation du contenu du dossier a du être menée de manière à disposer d'un modèle de dossier unique par discipline médicale [5]. Le même dossier peut être utilisé dans

\section{Résumé}

Alors qu'un dossier patient informatisé (DPI) est en place dans les établissements du Réseau Santé Valais / Hôpital du Valais depuis plusieurs années, le concept de système d'information sanitaire se déploie progressivement au sein du canton du Valais. Celui comprend outre le DPI, un serveur d'identité cantonal, ainsi qu'un datawarehouse contenant des données anonymisées indispensables pour piloter le système sanitaire valaisan. Les établissements médico-sociaux (EMS homes pour personnes âgées) et les centres médico-sociaux (CMS soins à domicile) disposeront également prochainement d'un DPI, alors que les médecins praticiens pourront recevoir et accéder électroniquement aux données médicales en provenance des hôpitaux et autres institutions.

les services de chirurgie de Martigny ou de Brigue. Outil de travail commun de l'ensemble des cliniciens (médecins, infirmiers, physiothérapeutes, pharmaciens et diététiciens), le DPI permet de documenter toute l'activité en lien avec le patient et autorise l'accès à l'information indépendamment des contraintes géographiques. Actuellement plus de 230000 dossiers hospitaliers comprenant entre autre 1100000 rapports sont disponibles. Les fonctionnalités générales du DPI sont résumées dans le tableau 1. Grâce à une base de données unique, il est facile pour l'utilisateur, sous réserve des droits d'accès, de pouvoir consulter les dossiers de séjours précédents au sein du RSV. Un mécanisme de copie de données permet de récupérer de l'information d'un séjour antérieur, comme par exemple des allergies ou des éléments d'une liste de problèmes médicaux, sans ressaisie manuelle par l'utilisateur.

\subsubsection{Serveur d'identité}

L'avènement des dossiers patients électroniques dans les différentes institutions de santé rend plus que nécessaire une identification univoque du patient afin d'assurer un accès exhaustif à l'information. Le traitement électronique des données d'identification du patient lors de son admission peut générer de multiples identifiants pour le même patient, que l'on peut appeler «doublons» de patient. Il en résulte une ressaisie de données ou une réutilisation d'anciennes données 


\section{Tableau 1}

Fonctionnalités générales du dossier patient informatisé de I’hôpital du Valais

Dossier médical: notes de suite, ordres, prescription de médicaments, rédaction de rapports et lettres de sortie

Dossier infirmier: documentation, processus de soins, feuille de surveillance, distribution des médicaments

Dossier médico-technique: physiothérapie, pharmacie, diététique

Système externes: résultats de laboratoire, rapports de pathologie, radiologie (rapport et images), rapport de coronarographie, tracé d'électrocardiogrammes ECG

Modules statistiques: anesthésiologie, actes opératoires, gynécologie-obstétrique

Aide à la gestion: gestion du bloc opératoire, gestion du flux des urgences

Figure 1

Projet INFOVAL, informatisation des hôpitaux valaisans.

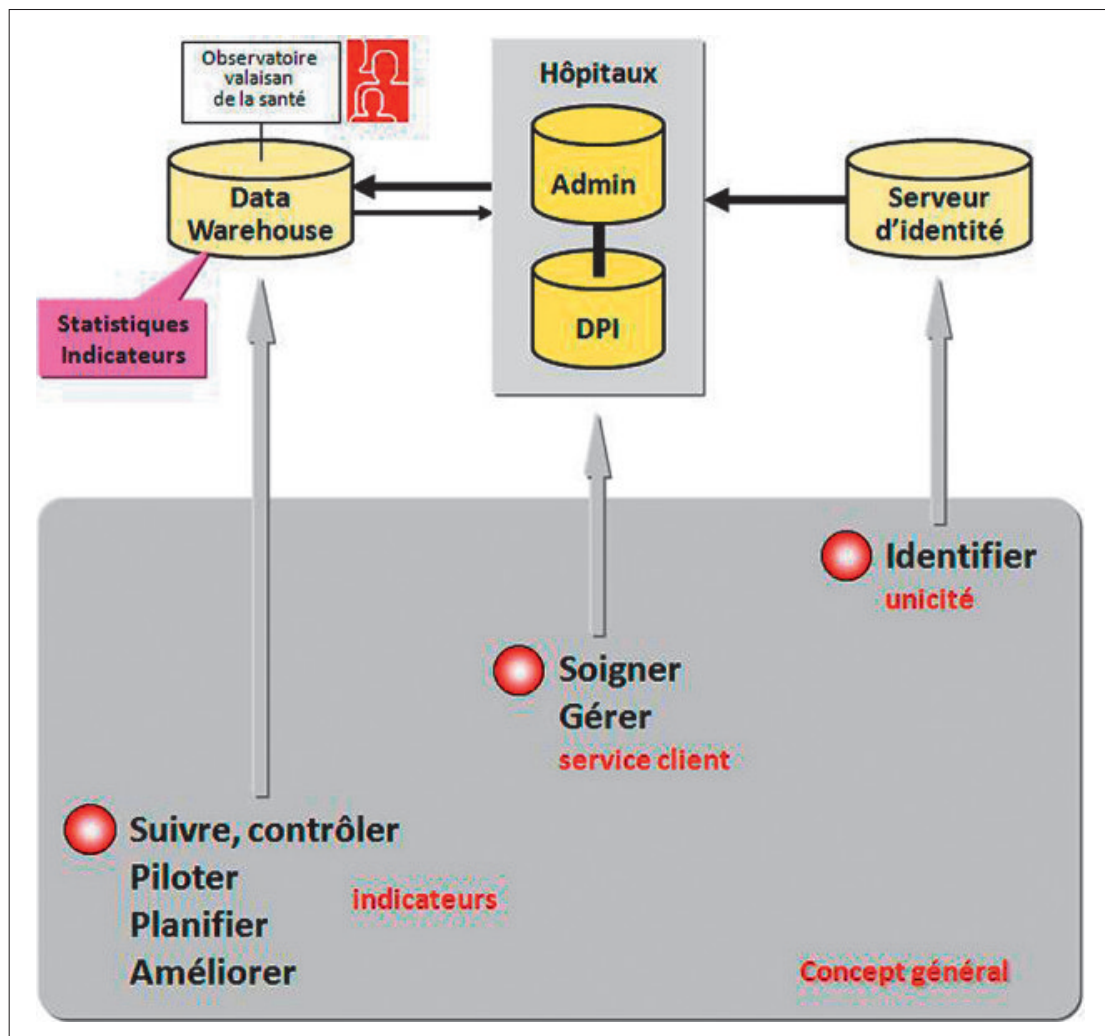

Figure 2

Extension du concept aux EMS/CMS.

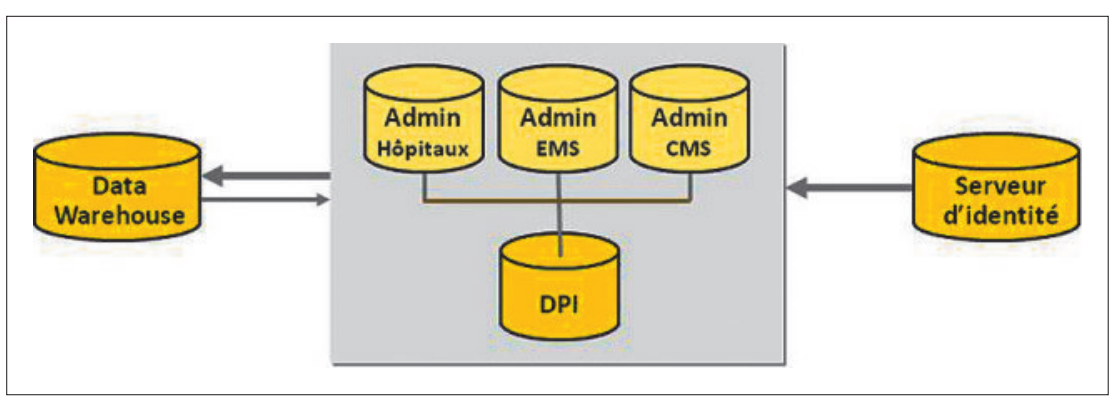

périmées (par exemple ancienne adresse) avec des coûts administratifs supplémentaires. Mais le problème principal concerne plutôt les données médicales avec une impossibilité d'accéder au dossier médical complet du patient.

Alors le nouveau numéro AVS (assurance vieillesse et survivant) permettrait de disposer d'un identifiant unique et immuable, diverses expertises juridiques mettent en doute son utilisation possible dans le domaine de la santé [6-8]. En outre le nombre important de patients étrangers, plus de 5000 durant l'année 2008 pour le RSV, et donc ne disposant pas d'un numéro AVS, rend d'autant plus nécessaire la disponibilité d'un outil d'identification unique. Pour ce faire, un serveur d'identité, ou Master Patient Index (MPI), capable de prévenir et détecter les doublons de patients a été implémenté. Il est basé sur un algorithme de phonétisation performant et prend en compte le nom, prénom, nom de jeune fille ainsi que la date de naissance du patient. Il a été conçu d'emblée pour pouvoir être intégré dans divers systèmes d'admission par le biais d'un service web.

\subsection{EMS-CMS}

Le projet Infoval a été poursuivi par un deuxième projet visant à regrouper (fig. 2), dans un même concept, l'ensemble des EMS (établissements médico-sociaux, homes pour personnes âgées) et des CMS (centres médico-sociaux, soins à domicile). Cette deuxième phase de réalisation a débuté en 2006 et devrait s'achever en 2011. L'intégration des EMS-CMS vient compléter la $1^{\text {re }}$ phase de réalisation en se reliant au même système d'information de soins (même logiciel DPI) ainsi qu'au serveur d'identité et au datawarehouse. Les applications administratives sont propres à chaque institution en raison de leurs spécificités respectives. L'emploi du même progiciel DPI que les hôpitaux permet de capitaliser sur les compétences acquises avec le projet des hôpitaux et favorise la réutilisation de formulaires lorsque ceci est relevant cliniquement. Comme pour les hôpitaux, une base de données unique hébergera les données du DPI de tous les EMS et CMS. Par contre l'option de créer une seule base de données commune avec les hôpitaux n'a pas été retenue pour de nombreuses raisons: le modèle des données ne sera pas identique, l'identifiant du patient ne sera pas commun car les systèmes administratifs maîtres des données seront différents, la taille de certaines tables de la base des données hôpitaux, et enfin la gestion des utilisateurs. En résumé la base de données des hôpitaux n'a pas été initialement conçue pour également héberger les données des EMS-CMS.

\subsection{Médecine ambulatoire, projet Infomed}

Ce $3^{\mathrm{e}}$ volet consiste à intégrer la médecine ambulatoire, privée et hospitalière, au concept de réalisation d'un système d'information sanitaire intégré et unique au canton du Valais. Il s'agira ainsi d'aborder cette étape en considérant 3 cas de figures distincts, condui- 
sant à des solutions logiques et physiques différentes, soit:

- la médecine ambulatoire hospitalière, par exemple: les urgences, les opérations ambulatoires, les chimiothérapies, les radiothérapies, les dialyses, ...;

- la médecine ambulatoire privée constituée de médecins libéraux. Dans ce cas spécifique, une attention particulière sera portée aux relations du médecin traitant avec ses patients hospitalisés ou soignés à domicile (CMS) ainsi qu'aux médecins répondants des EMS;

- la médecine ambulatoire «mixte» concernant des médecins, généralement spécialistes, exerçant en médecine libérale (cabinet privé) et hospitalière (par exemple les gynécologues et les chirurgiens).

Actuellement, la proportion des médecins privés disposant et utilisant un DPI est faible. Une étude récente estime à un peu plus de $10 \%$ le nombre de cabinets privés équipés d'une solution informatique de suivi des dossiers patients [9]. Dans ces 10\% d'informatisation des soins, les solutions actuellement mises en place sont diverses, de fournisseurs différents, donc avec un degré d'harmonisation et d'intégration faible, et des pratiques d'utilisation variées.

La pratique de la médecine ambulatoire étant libérale, il n'est pas possible, ni forcément souhaitable, en l'état et pour la gestion des données médicales du médecin, d'imposer un système ou un logiciel informatique. Il est dès lors prévu de proposer et offrir aux médecins privés la possibilité de visualiser, par une solution informatique légère (site internet sécurisé), le suivi des dossiers de leurs patients hospitalisés. En

Figure 3

Flux des données médicales et d'identification du patient. CDA-CH: Spécification pour l'échange électronique de documents médicaux en Suisse.

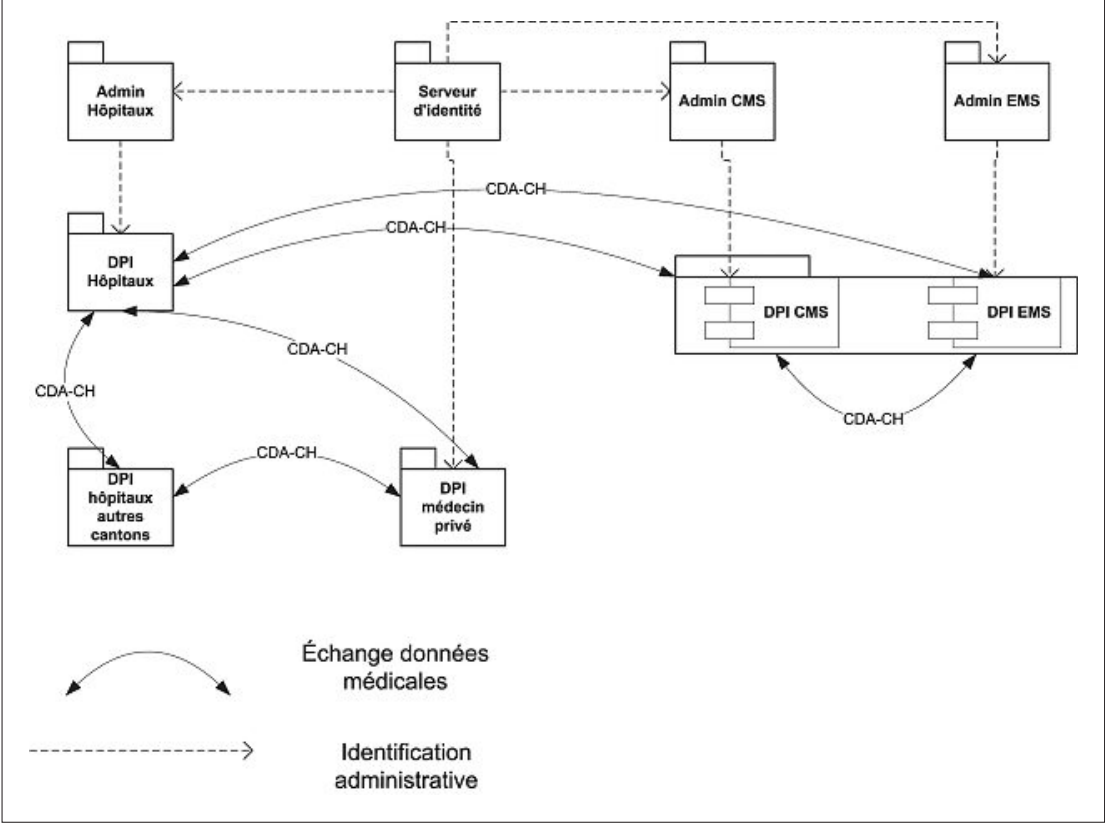

parallèle il leur sera proposé un transfert informatique, en fin de traitement hospitalier, de la lettre de sortie de leur patient et d'informations médicales plus détaillées nécessaires à la suite du traitement posthospitalier. Ce transfert se fera selon des standards internationaux qui devraient être reconnus par les applicatifs DPI de chaque médecin, quelque soit le choix du fournisseur. Une liste de logiciels recommandés et compatibles avec ces standards devrait ainsi être établie.

Pour les médecins répondants des EMS il leur sera possible d'intervenir à distance, en toute sécurité et moyennant toutes les garanties d'authentification, sur le DPI de l'établissement. Une procédure identique sera également envisagée dans le cadre des CMS.

En ce qui concerne la médecine ambulatoire hospitalière, l'utilisation du DPI hospitalier est retenue mais nécessitera des adaptations aux pratiques et exigences particulières de ces activités de soins.

La stratégie proposée pour l'adhésion des médecins privés au projet est basée sur une approche d'offre et de mise à disposition de l'information. La démarche est alors basée sur une approche en «flux poussé» de données médicales, c'est-à-dire la possibilité, pour les médecins privés et en première approche, de recevoir les données de soins de leurs patients hospitalisés (séjour ou ambulatoire) directement depuis le DPI hospitalier. Cette approche devrait susciter le développement des DPI auprès des médecins privés et créer, de manière informatique fiable et sécurisée, la synergie du partage et de l'échange des données médicales du patient tout au long de sa vie, quelques soient les traitements qu'il a subi et les établissements qu'il a fréquenté.

Une première expérience d'échange de données médicales électroniques a pu être obtenue dans le cadre du projet Medicoordination auquel participe le RSV. Le projet est initié par la Haute Ecole de Suisse Occidentale HES-SO (Sierre et Fribourg) et vise à échanger électroniquement des avis de sortie entre les hôpitaux et les cabinets médicaux. Des informations supplémentaires sur ce projet sont disponibles sous http://www.medicoordination.ch

\section{Echanges de données et respects des recommandations cybersanté de la Confédération}

Si l'architecture des différents systèmes d'information clinique a pu être décrite ci-dessus, il n'en reste pas moins qu'il est nécessaire de définir les modalités pratiques d'échange des données. Nous pourrons pour ce faire nous appuyer sur le standard CDA-CH(10) élaboré par le groupe HL7 Suisse. Ce même standard sera utilisé non seulement pour exporter des données médicales vers les partenaires non directement intégrés dans le système d'information sanitaire du Valais, comme par exemple les hôpitaux universitaires, mais également à l'intérieur du système d'information sanitaire valaisan. Comme on l'a vu les DPI des hôpitaux et des EMS-CMS sont basés sur le même logiciel, mais au lieu de développer des protocoles propriétaires 
d'échange de données, nous souhaitons d'emblée respecter le standard CDA-CH (fig. 3).

L'échange électronique de données médicales nécessite également une identification précise des patients et des intervenants. Les fournisseurs de prestations seront identifiés par l'intermédiaire du numéro GLN (anciennement EAN) enregistré dans la base MedReg [11]. Les autorisations d'accès des médecins praticiens s'effectueront par l'intermédiaire de la nouvelle carte professionnelle de santé [12] émise par la Fédération des Médecins Suisse. L'identification des patients s'appuiera sur le serveur d'identité cantonal préalablement décrit. Grâce à celui-ci, nous espérons pouvoir éviter l'utilisation de la carte d'assuré, dont l'emploi nécessiterait une loi cantonale [13] et dont les différentes instanciations par les assureurs ne facilitent pas son utilisation. L'approche «flux poussé» de données médicales évite, dans un premier temps, la nécessité de création d'un registre des documents (repository) avec tous les problèmes légaux et techniques associés.

De manière générale nous pouvons ainsi affirmer que les recommandations du projet partiel «Normes et architectures» [14] de la stratégie cybersanté de la Confédération devraient ainsi être pleinement respectées.

\section{Conclusions}

L'évolution du concept d'informatisation des données de soins de l'ensemble des partenaires de la santé du canton du Valais devra permettre (fig. 4):

\section{Figure 4}

Evolution du concept intégré de données liées aux soins.

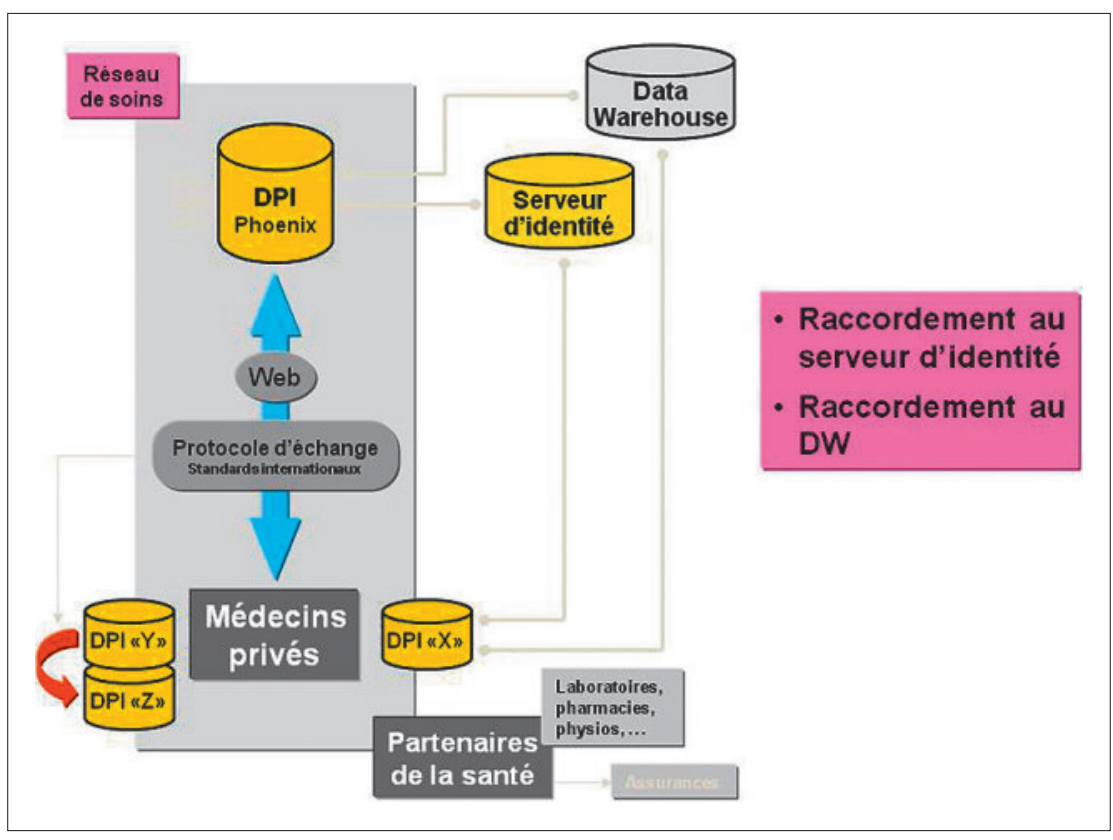

- l'interopérabilité des données en privilégiant les transmissions de données médicales selon des protocoles sécurisés respectant les standards internationaux et les recommandations de la stratégie cybersanté de la Confédération;

- le rattachement de toutes les bases de données de soins (DPI des médecins privés et autres partenaires de la santé) au serveur d'identité cantonal et au datawarehouse.

\section{Références}

1 Paccaud F. Statistique sanitaire en Valais: proposition pour un observatoire cantonal (Internet). 1999. (cité 2009 Nov 28). www.obs-vs-sante.ch/ documents/proposition.pdf

2 Wieser P. Informatisation des hôpitaux valaisans (Internet. Observatoire valaisan de la santé; 2000. (cité 2009 Nov 28) www.obs-vs-sante.ch/documents/ Rapport_Wieser_F.pdf

3 Observatoire valaisan de la santé/Walliser Gesundheitsobservatorium (Internet). (cité 2009 Déc 4). www.ovs.ch/

4 Gnaegi A, Cohen P, Marey D, Rivron M, Wieser P. Satisfaction des utilisateurs du dossier patient informatisé valaisan. Swiss Medical Informatics. 2006;(59):6-8.

5 Cohen P, Gnaegi A. Démarche de standardisation des documents de travail des hôpitaux dans la construction d'un système d'information clinique. Swiss Medical Informatics. 2006;(59):32-5.

6 Walter J. Vers une société sous surveillance? La problématique du nouveau numéro AVS (Internet). (cité 2009 Déc 9). http://ditwww.epfl.ch/SIC/ SA/SPIP/Publications/IMG/pdf/sp-06-page15.pdf

7 Giger M. Le «patient transparent». Bull Méd Suisses. 2007;88(38):1575.

8 Schwalm B. Methode zur Vereinfachung einer datenschutzrechtlichen Analyse von Prozessen in eHealth. Swiss Medical Informatics. 2010;(68):31-3.

9 Schumacher M. MediCoordination. Clinicum Romandie. 2009;1(1):17-9.

10 Hanselmann M, Knoepfel C, Schaller T, Steiner P. CDA-CH: Spécification pour l'échange électronique de documents médicaux en Suisse (Internet); 2009. (cité 2009 Déc 9). www.hl7.ch/default. asp?tab=2\&item=standard

11 Hodel M. Le registre des professions médicales (registre). Bull Méd Suisses. 2009;90(48):1875-7.

12 Carte de professionnel de santé CPS/HPC (Internet). (cité 2009 Déc 10). www.fmh.ch/fr/services/cps_hpc. html

13 Ordonnance sur la carte d'assuré pour l'assurance obligatoire des soins. 2007.

14 Cybersanté Suisse, Normes et architecture, Premières recommandations (Internet). ehealthsuisse; 2009. (cité 2009 Déc 9). www.e-health-suisse.ch/umsetzung/ 00092/00096/index.html?lang=fr 